\title{
Topic, Focus and The Structural Dynamics of Language*
}

\author{
Ruth Kempson, Ronnie Cann, Jieun Kiaer \\ King's College London \\ \{kempson, kiaer\}@dcs.kcl.ac.uk, r.cann@ed.ac.uk
}

December 29, 2003

\begin{abstract}
The characterisation of a grammar formalism for natural languages defined in terms of the process of time-linear tree growth for interpretation explains syntactic topic and focus effects at the left and right periphery as consequences of basic tree growth processes, much as Vallduvi 1991, but without positing any additional level of structural representation.
\end{abstract}

\section{Preliminaries}

The concepts of topic and focus involve a context-sensitivity that uneasily straddles the boundaries between syntax, semantics, and pragmatics. ${ }^{1}$ In accounts of the phenomena in the principles and parameters and minimalist frameworks, topic and focus are both treated as associated with one or more projected functional heads (see Rizzi 1997 and others), with no attempt to explain the context-sensitivity of the phenomena. Model-theoretic approaches, to the contrary, address their context-dependence through questionanswer congruences (eg Büring 1997, Schwarzschild 1999), but they do not provide a basis for expressing restrictions on syntactic forms of focussing device, and, despite context-dependency in the explanation, their accounts fail to allow any shift of focus in providing an answer to a question. Vallduvi

*This paper reports a team effort in developing a new framework. Thanks to all, especially Wilfried Meyer-Viol, Masayuki Otsuka, Lutz Marten, Eleni Gregoromichelaki.

${ }^{1}$ There are number of overlapping distinctions: topic vs focus, given vs new, presupposition vs focus, topic vs comment, theme vs rheme (see Sgall et al 1986, Halliday 1967, Chomsky 1971, Vallduvi 1991, Erteschek-Shir 1997. 
(1991) and Erteschek-Shir (1997) claim to capture structural and semantic aspects the construal of topic/focus by articulating an additional level of Information Structure, but this level lacks independent motivation and implies a non-minimal architecture for the grammar.

In this paper, we argue that the Dynamic Syntax framework (DS) which adopts a Fodorian representationalist stance, ${ }^{2}$ with the added twist of reflecting the dynamics of real-time parsing - is able to articulate structural processes of interpretation build-up, from which the reported topic and focus effects can be explained. The account to be set out expresses similar insights to Vallduvi and Erteschek-Shir, but in a framework in which the dynamics of the parsing process constitute the grammar formalism and provide all that is needed to explain these effects.

\section{The Dynamics of Language Processing}

The methodology implicit in the Dynamic Syntax formalism is to take the constructs used in semantics, and define them as representations for which a syntax is defined. The process of establishing some such interpretation in context is defined as a monotonic tree growth process. ${ }^{3}$ The central claim is that syntactic properties of natural language resides exclusively in the progressive growth of such tree-structure representations strictly following the dynamics of left-right processing. ${ }^{4}$ No additional level of syntax needed.

The type of tree we assume as interpretation assigned to (1) is (1'):

(1) John upset Mary

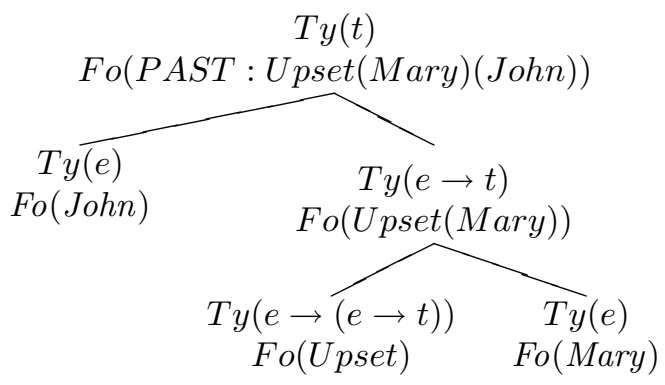

\footnotetext{
${ }^{2}$ There is a long and, by now, respectable tradition in cognitive psychology (Fodor 1981 and others following) that human reasoning, being mind-internal, is essentially syntactic, that is, defined in terms of licensed moves from one representation to another purely in virtue of form.

${ }^{3}$ With all content represented in a tree-structure format, the context against which utterance interpretation takes place is also represented as a set of trees.

${ }^{4}$ More strictly, processing is time-linear. The term time-linear is due to Hausser (1989).
} 
Each node in the tree has a concept formula (Fo for Formula) and an indication of what semantic type that concept is. The primitive types are types $e$ and $t$. All other types are functions on these. ${ }^{5}$ All this is no more than a conventional semantic characterisation of how sentences are understood in context as assertions about entities in the world around us, expressed as a predicate-argument formula, with the tree showing how the variously typed expressions that are sub-terms of the containing formula combine together to yield that resulting formula - in effect a history of how the parts combine together.

\subsection{Building up Structures}

Secondly, the trees are not induced from the lexicon on a head-driven basis, but as an incremental parsing device, building up a sequence of partial trees, each one an enrichment of the previous partial tree in the sequence. The overall dynamics is to unfold a skeletal structure in anticipation of concepts which the words progressively provide in the order in which they are presented in the string, and then to combine those concepts together on a strictly bottom-up basis to yield a propositional formula. ${ }^{6}$ The starting point, associated with the outset of the parsing process is simply the goal to establish some propositional formula as interpretation, stated as ?Ty(t); ${ }^{7}$ and this overall goal may lead to other subgoals as more information comes in, these represented as additional nodes in the tree, eg with requirements ?Ty $(e), ? T y(e \rightarrow t)$. In this respect the process is top-down. The progressive building up of interpretation is taken to be a process of unfolding a tree structure in anticipation of what is needed to complete a logical form representing an interpretation using all the words, these words providing actions that enable appropriately decorated structure to be built up (the

\footnotetext{
${ }^{5}$ The Formula values that decorate the nodes are not words of the natural language. In describing their associated types, type $e$ is for expressions that denote individuals ( $e$ for "entity"); type $t$ is for propositional formulae, that denote truth values ( $t$ for "truthvalue"). One-place predicates are functions from individuals to truth-values - they combine with argument expressions to give a propositional formula - so they are of type $(e \rightarrow t)$. Two-place predicates are expressions which combine with an argument expression to yield a one-place predicate - i.e. of type $(e \rightarrow(e \rightarrow t))$. See Kempson et al 2001 for a detailed formulation.

${ }^{6}$ The formal core of the framework is the Logic of Finite Trees (Blackburn and MeyerViol 1994), in which $\langle\uparrow\rangle X$ is defined from node $n$ as " $\mathrm{X}$ holds at the mother of $\mathrm{n}$ ", $\langle\downarrow\rangle X$ from node $n$ as $\mathrm{X}$ holds at a daughter of $n$.

${ }^{7}$ The decision to model this task as goal-directed follows that of Relevance Theory: Sperber and Wilson 1995. Indeed the impetus for this framework was to provide an architecture within which study of pragmatic constraints could be given a formal setting.
} 
words providing the bottom-up aspect of the parsing process).$^{8}$

\section{$2.2 \quad$ Reflecting context-dependence}

Pursuing the representationalist stance, all context-dependent aspects of interpretation are represented by underspecified terms to be replaced by substitution of terms as the unfolding structure makes them available. So a pronoun is defined as providing a placing-holding device, a metavariable, which has to be replaced during the interpretation process by some OTHER term as made available in the context. For example, in (2), he and her are naturally understood as picking out John and Mary by being replaced by the forms $F_{o}\left(J_{o h n}\right)$ and $F o($ Mary $)$ :

(2) John ignored Mary. He upset her.

The concept of the context-dependence of anaphoric expressions in language is familiar enough. ${ }^{9}$ What is less orthodox is the assumption that it is to be defined as a tree-update process; and with contexts also represented as (partial) trees, anaphora resolution can apply equally to the update of a pronoun from antecedent terms within the structure under construction:

(3) John thinks that he is clever

For the identification of he in (3), the context relative to which that interpretation process takes place includes the partial structure containing the subject node with its decorations.

In canonical uses of pronouns, like all other content words, the pronoun has a restriction that whatever value it is assigned must be taken as decorating a terminal node in the tree. ${ }^{10}$ As we shall shortly see, there is a range of expletive pronoun uses in which this terminal node restriction is lost.

\subsection{The dynamics of long-distance dependency}

What is novel is the extension of this concept of underspecification and update during the interpretation process to the modelling of long-distance dependency effects:

\footnotetext{
${ }^{8}$ In any partial tree, there is one node indicated by a pointer, $\diamond$, as the node under development. In this framework all noun phrase construals are taken to be of type $e$, matching arbitrary names manipulated in natural-deduction proofs. Accordingly, the terms onto which words map are lambda terms within the epsilon calculus (the epsilon calculus provides the formal study of arbitrary names).

${ }^{9}$ See eg Ranta 1994 for an account of anaphora resolution as variable substitution.

${ }^{10}$ By definition, words decorate terminal nodes in a tree, a so-called "bottom" restriction, in addition to whatever other tree-update actions they may induce.
} 
(4) Mary, John upset.

Structures such as these have been universally assumed since Chomsky 1965 to display a core syntactic phenomenon not reducible to semantic explanation. The present analysis, however, does not set up such separate syntactic mechanisms, but, instead, follows the parsing dynamics. An expression introduced at an early stage in the interpretation process does not, at this point in the interpretation process, enable its position in the structure to be identified. In (4), the word Mary is construed as providing a term for the resulting logical form, but the node which it decorates does not have its relation within the overall structure yet fixed: ${ }^{11}$

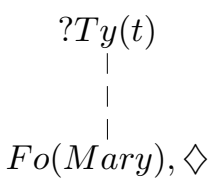

This is fixed later, following an arbitrary number of intermediate steps in the interpretation process. It is only after processing the word upset, that the opportunity to provide this fixed position will arise. At that juncture, there will be a structure with the concept $F o$ (Upset) labelling a functor node, and a node introduced to provide its internal argument:

(c) After processing upset:

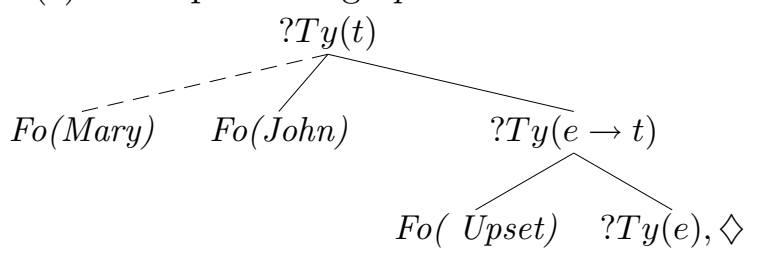

Both the type requirement of the object node and the requirement for a fixed position in the tree for Fo(Mary) drive the subsequent step, which is to unify these two nodes. Nothing more will be needed if the role of the formula Mary is identified as being the object of Upset, for such a move

\footnotetext{
${ }^{11}$ Formally, this is defined using the Kleene star operation defined on the daughtermother relation: $\left\langle\uparrow_{*}\right\rangle \operatorname{Tn}(a)$ is a node dominated by some node $T n(a)$. Adding the requirement ? $\exists x . \operatorname{Tn}(x)$ as an additional decoration on that node imposes the requirement that in all successful completions of the tree, this underspecified characterisation, in effect a disjunction across all possible sequence of mother relations from that node to $\operatorname{Tn}(a)$, is replaced by a fixed tree relation. See Kaplan and Zaenen 1989 for use of the Kleene * in defining 'functional uncertainty' in LFG, equally an account of long-distance dependency. The difference between this and the Kaplan and Zaenen account lies in the concept of growth articulated in Dynamic Syntax as part of the construction process.
} 
will simultaneously satisfy the two outstanding terminal-node requirements. From then on, the process is one of combining the concepts Fo(Upset) and Fo(Mary), and then the predicate $F_{O}(U p s e t(M a r y))$ and Fo(John) to yield the logical form $F_{0}$ (Upset(Mary)(John)), with a resulting tree identical to that derived from the parse of (1).

This already provides us with a means of identifying what might be involved in a focussing device. Defined as it is in terms of left-right processing and progressive update of structure, the first constituent is initially analysed as providing some information, but not the role of that term in the structure. Its structural role is thus initially underspecified and resolved later when some appropriate structure has been introduced. The dynamics of this process, accordingly, involves the isolation of one term at the node in question and the immediately subsequent construction of an accompanying propositional structure, to which that one term provides a subsequent update. The concept of syntactic focus, we shall be suggesting, is no more than this - the provision of some structure identified as providing the update to some constructed structure that yields a completed propositional formula. ${ }^{12}$

\subsection{Constructing trees in tandem}

In addition to the projection of such individual trees, paired linked trees are defined. One partial tree, that is, may provide the context for the process of introducing another, so that two trees are, in effect, introduced in tandem, using a combination of anaphoric substitution processes across a pair of trees and mechanisms for updating an individual tree structure. Relative clause construal provides a core example:

(5) John, who left, cried.

Following the dynamics of how such trees might be incrementally constructed, in the DS account, a LINK transition is defined (using an additional modal operator $\langle L\rangle$ and its inverse $\left\langle L^{-1}\right\rangle$ ) that licenses a transition from a node in one tree to the initiation of a second. Expressed as a modal requirement on the way this new emergent tree is to be completed, the tree is constrained so that at some point in its subsequent construction, there must be a copy of the formula from the head from which the LINK relation was constructed. ${ }^{13}$ In (5), that relation is constructed from the node deco-

\footnotetext{
${ }^{12}$ See Kiaer in preparation for a defence of the view that this explanation extends to phonological stress.

${ }^{13}$ Formally, this involves the combination of the LINK transition defined as introducing a LINK relation from the head node decorated with some term $F o(\alpha)$ to a new type-t-
} 
rated by $F_{O}\left(J_{o h n}\right),{ }^{14}$ with the copy being provided at an unfixed node by the interpretation assigned to the relative pronoun (Fig.1).

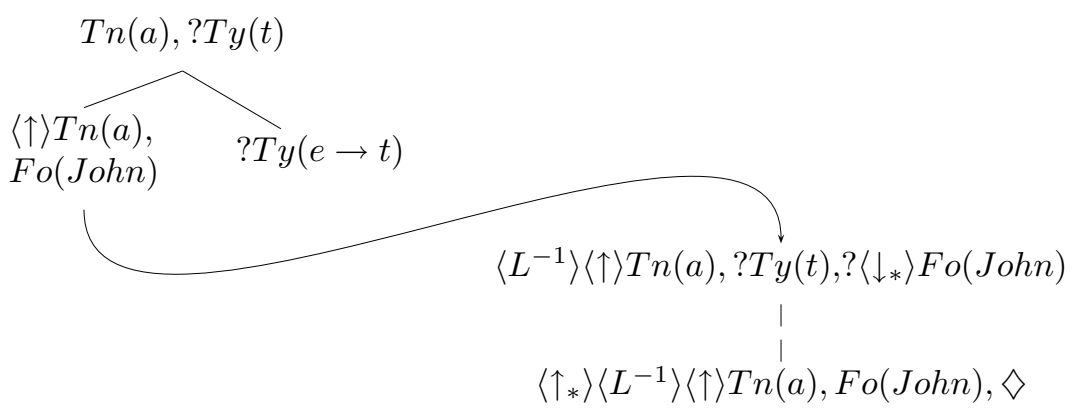

Figure 1: Parsing who in (5)

\subsubsection{Linked structures from left-peripheral expressions}

This form of relation between obligatorily anaphorically paired trees applies naturally to account for Hanging Topic Left Dislocation structures (Anagnastopoulou et al 1997), for nothing in the formal statement of the rule licensing the LINK transition dictates the level of embedding that the head node might be at in its containing structure; and nothing prevents it being the top node of some structure. Accordingly, we propose that the leftperipheral expression may be used as the trigger for introducing a tree linked to the rootnode which is to be of type $e$ which that expression duly decorates (Fig.2). The consequence of having constructed and decorated such a linked tree is that the root node with requirement ?Ty $(t)$ must now be constrained to contain a copy of the term projected from the left-peripheral noun phrase so as to satisfy the sharing-of-terms requirement dictated by the LINK relation between the two structures. This modal form of requirement determines the presence of a suitably construed pronoun in the twinned structure, which the facts of so-called hanging topic left-dislocation corroborate:

requiring node, and the added requirement ? $\left\langle\downarrow_{*}\right\rangle F o(\alpha)$ - viz. the requirement that some daughter node to this top node must be decorated with a copy of the very same term that decorates the head from which the LINK transition was built.

${ }^{14}$ Nodes decorated with type $e$ formulae may have internal structure containing a variable-binding term-operator which is the quantifying device, a variable also of type $e$, and a restrictor. Restrictive relative construal involves defining a LINK transition from the node decorated by the variable. Nonrestrictive relative construal involves a transition from the top-node of this structure, also of type $e$. In a fuller characterisation of naturallanguage names, these would be analysed as iota terms built up from such sub-structures. 


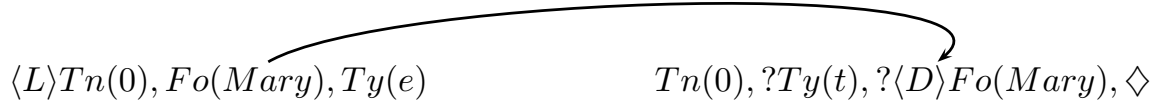

Figure 2: Building a linked structure for left-peripheral expressions

(6) As for Mary, Tom adores her

(7) *As for Mary, Tom adores.

The form of the requirement involves the modal operator $\langle D\rangle$, which is the union of LINK and daughter relations, i.e. embodying a restriction that there be some subsequently constructed copy of the head term, without any structural restriction on where in the structure such a copy should occur:
(8) I Maria xtes gnorisa ton andra pu tin The Maria ${ }_{N O M}$, yesterday I met the man who her patreftike.
[Greek $]$ married

'As for Maria, yesterday I met the man who married her.'

The only exception to this required presence of a lexical pronoun is the case of pro-drop structures, i.e. those cases where the verb projects the its argument nodes decorated with a metavariable, exactly as though a lexical form of pronoun had been present.

\section{Characterising the left periphery}

We can now see how, with these two mechanisms for projecting interpretation from left-peripheral expressions, we can define their correspondence with various topic and focus effects.

It has been standardly recognised over a long period (Ross 1967) that long-distance dependency effects can be modelled either as a correlation between two discrete positions in a tree through a process such as movement (or feature-passing), or through a process of anaphoric linkage. There are languages with the left-dislocated expression paired with 'a gap' and displaying island restrictions, a diagnostic of movement processes:

(9) Mary, John thinks Tom had upset. 
(10) *Mary, I dislike the man that married.

These are reconstructed here as initially unfixed nodes, updated at some point along a sequence of daughter relations, by definition excluding resolution in all structures analysed as projecting linked trees - that is relative clauses, coordination and clausal adjuncts. There are also languages/structures that display pairing of the left-dislocated expression with a pronoun with no subjacency effects; and these have been assumed to be induced as independent structures - linked to each other solely through anaphoric processes. These correspond straightforwardly with the concept of linked structures:

(11) Il-kita:b da, 'inta tkallimt ma9a l-walad 'illi katab the book this, you talked with the boy who wrote

galey-h.

[Egyptian Arabic] on it

'You talked with the boy who wrote on this book'

(12) As for Mary, I talked to the boy who had scribbled on her book.

What is less expected, given this dichotomy, are the various intermediate forms, involving some kind of interaction between the establishment of a long-distance dependency effect and anaphoric processes, and an apparent blurring of the distinctiveness in the two processes. There are left-peripheral constituents paired with a pronoun which display some but not all properties of movement - their properties include sensitivity to strong island effects, general exclusion of quantified expressions (indefinites only, and with specific interpretation), being associated with a sharp break of intonation following the left-peripheral expression: ${ }^{15}$

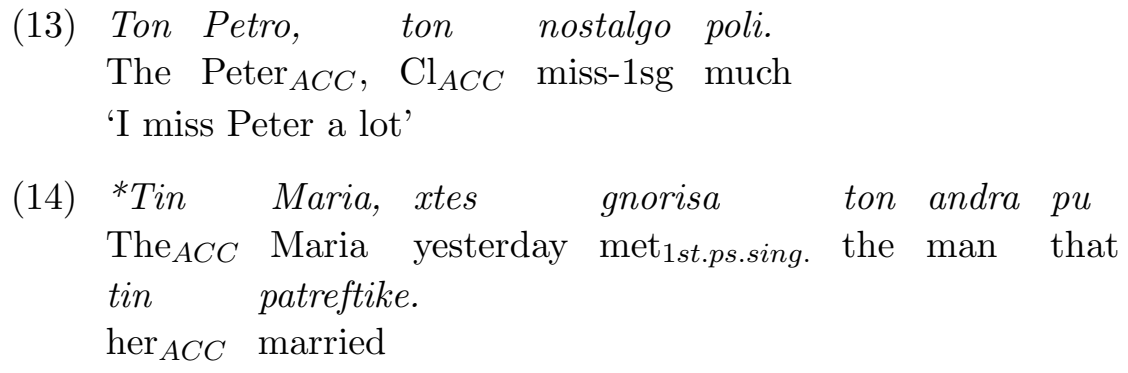

\footnotetext{
${ }^{15}$ As a construction type, this was first identified as Clitic Left Dislocation (CLLD) by Cinque 1991, who argued that despite their sensitivity to strong island effects, these data nevertheless required a base generation account. This range of variation is widespread. For example, Aissen 1992 has argued that the Mayan languages differ according to whether they have an external topic which is an independent structure, separated by an intonational break, or have no such break but are able to occur in subordinate clauses.
} 
Mary, yesterday I met the man that her married'

These intermediate effects are problematic for movement accounts, ${ }^{16}$ as the paradigm leads one to expect a certain diagnostic set of effects associated with movement, and failure to meet such diagnostics is associated with base generated pairing of coindexed expressions. The two theoretical constructs have been taken to be discrete; and the existence of Clitic Left Dislocation, since its identification in Cinque 1991, is generally taken to require base generation of the left-peripheral expression and its co-indexed clitic, despite meeting many of the diagnostics of movement. ${ }^{17}$

\subsection{Linked trees as constructed contexts}

The advantage of expressing pronoun construal and long-distance dependency in the same terms of underspecification and resolution through tree growth is the natural characterisation it allows for intermediate effects. They can arise in one of two ways: either through the imposition of locality constraints on the antecedent-anaphor relation imposed by the LINK relation, or through the feeding relation between pronoun construal and long-distance dependency, a node decorated by a pronoun being able to be unified with some unfixed node and so receiving a value through the Merge process as an alternative form of update for pronoun construal.

Taking up the form of restriction dictating how two structures are to be taken as linked, in our prototype display, figure.2, we took the form of requirement to be ? $\langle D\rangle F o(\alpha)$, indicating that there were no locality constraints on where the copy was to be constructed within the individual tree on which this requirement was to be met. However, given the modal form of the requirement, there is every reason to expect variants of this form of constraint through selection of other modal operators from the range $\langle D\rangle,\left\langle\downarrow_{*}\right\rangle$, thereby imposing more severe restrictions on where the copy is to be found in this second tree. The imposition of the requirement using the $\left\langle\downarrow_{*}\right\rangle$ operator, where the imposed structural restriction on the antecedent-anaphoric pairing mimics that of an unfixed node, corresponds directly to CLLD structures: and, given the LINK analysis, the attendant pronoun in the second structure and sharp intonational break of CLLD are also expected. ${ }^{18}$

\footnotetext{
${ }^{16}$ There may even be variation between forms within a single language, as in Romanian, which has one left-dislocation structure associated with one kind of morphological marker, which displays no island restrictions, a second which does.

${ }^{17}$ The difficulty in sustaining this dichotomy is increasingly leading to analyses which depart from it (see Adger and Ramchand forthcoming).

${ }^{18} \mathrm{On}$ this analysis, one might expect further that if a language had morphological means
} 
The advantage of this approach is not merely in providing a basis for articulating systematic interactions between anaphora and island-inducing structures/processes. It also confirms the correspondence between topic, context and linked tree. From the concept of a pair of linked trees, we can define topic as a direct reflection of a particular form of context, that of a partial tree constructed during a single utterance process. At its most general, then, topic is a structure that provides an antecedent for later identification by some anaphoric device. This may be on a cross-utterance basis:

(15) John came in. He was sick.

But it may take the form of some constructed linked tree as the context which forms a basis for antecedenthood for other terms introduced as part of the process of interpretation for the string, as in Hanging Topic structures:

(16) As for John, he was sick.

Left-peripheral expressions construed like this may or may not be anaphorically identified from the more general context. Contrastic topics arise when the linked structure so constructed is NOT itself anaphorically linked to the general context, and in so being a departure from it, will be in contrast to it. No structural distinction is needed to reflect this: the relation of a pair of linked structures to the more general discourse context is sufficient.

\section{$3.2 *$ Adjunction and the basis of focus}

We now turn to the second way in which nodes in a tree may be correlated - through the projection of an unfixed node and its subsequent merge with a distinct node in the tree. Here we find the inverse effect. One term may be isolated from some remainder, because, in virtue of its isolatability, it can be used as an update for a structure once that structure is substantially complete. And once again, there are ways in which structural processes yielding this result interact with anaphora construal to yield mixed effects.

The core cases are when an unfixed node can be decorated and left without a fully determined structural relation until later in the construction process. By definition, this is the building of one node within a structure whose position is not fully determined, so that update must be within that structure, precluding the possibility of a weaker restriction, that the update

of distinguishing more than one type of such structure, these might differentiate between different forms of locality constraint, yielding the type of variation observed in Romanian. 
could be across paired structures. However, a form of update for (17) is also available in which an unfixed node within a SINGLE structure can be updated by merging it with the node decorated by that pronoun itself:

$$
\begin{aligned}
& \text { Ti Maria }{ }_{A c c} \text { (ti) sinantise xtes } \\
& \text { the Maria, (her) I met yesterday }
\end{aligned}
$$

[Greek]

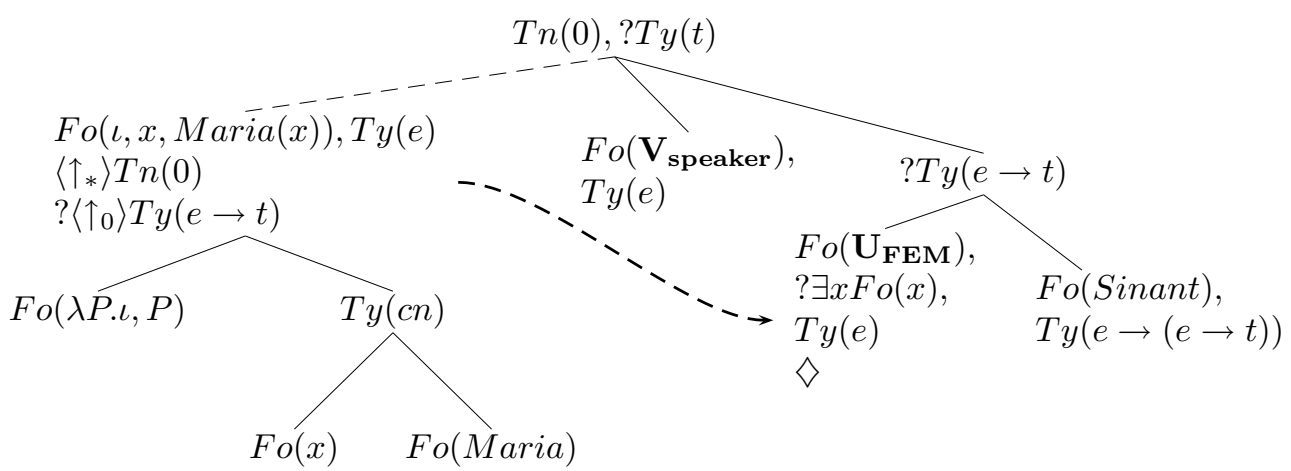

Figure 3: Licensing pronoun-merge interaction

Whether this form of update is possible turns on whether the pronoun lexically imposes a terminal-node restriction. If it does, it will not allow such a process (retention of the terminal-node restriction is characteristic of English). ${ }^{19}$ If it does not, such an alternative is possible, allowing it to decorate a node which might turn out to be nonterminal in the resulting tree. In Greek, with its possible matching of case-marking on the clitic pronoun and left-peripheral expression, such an analysis appears to be required. ${ }^{20}$

In standard Spanish, such an analysis appears to be essential to distinguish the dative clitic pronoun, as, out of all the clitics, it alone can be used

\footnotetext{
${ }^{19}$ The only exception is expletive $i t$, paired with propositional formulae (see section 4.2 ). English relative clauses, in which resumptive use of personal pronouns is an available, albeit marked option, do not provide evidence of any such loss of restriction as in these LINKed structures, it is only the formula value that is copied over into the LINKed structure, and not any ancillary structure (see Cann, Kaplan and Kempson 2004).

${ }^{20}$ Object clitic pronouns in Greek are standardly analysed as an object agreement marker. The idiosyncratic position of clitics before the verb, which is problematic in other frameworks in occurring in positions in which full noun phrases may not, is here analysed as a lexical calcification of what in the common root to the modern Romance languages, Latin, was a free process of a localised form of *Adjunction which, by the licence of introducing an unfixed node whose position in some local predicate-argument structure is immediately determined by case, enables argument nodes to be processed in any order (see Kempson 2003 for an account of Japanese short scrambling in these terms).
} 
to duplicate ALL forms of NP, quantified and referential:

(18) A nadie le devolvió Maria su manuscrito [Spanish] To no-one $\mathrm{CL}_{D A T}$ returned Maria his manuscript.

'To no-one did Maria return his manuscript'

(19) A familias de pocos medios les ofrecieron queso y to families of small means to them offer $3 p l$ cheese and leche milk

To low-income families, they offered cheese and milk'.

All other clitic doubling involves a specificity restriction indicative of a pair of linked trees established through the anaphoric linkage: ${ }^{21}$

(20) un coche Maria lo compró

[Spanish]

a car Maria it bought

'A particular car Maria bought it'

Further confirmation of the analysis comes from the ambiguity of construal of Spanish subjects. Given the lack of morphological marking of case on the subject, and the pro-drop properties of the verb, projecting a full template of structure and a placeholder in subject position able to be identified contextually, we correctly anticipate that the subject may or may not be construed contrastively. ${ }^{22}$

This account of clitic-duplicated left-dislocation structures matches the more familiar base generation vs movement accounts in many respects, but it has the edge over them in that the intermediate cases emerge as expected variants, either from the interaction of independently motivated processes, or as expected variations along a locality spectrum. ${ }^{23}$ In particular, the various anaphoricity effects that are achieved do not need to be formalised with

\footnotetext{
${ }^{21}$ The specificity arises because scope is defined relative to individual propositional structures, having been established incrementally during the parse process: indefinite noun phrases are the only apparent exception, because their scope can be indefinitely extended (see Kempson et al 2001, ch.7). The Rio Platense accusative clitic appears to be moving in the direction of the dative, though retaining a specificity restriction on all doubling of the clitic. See Suñer 1988.

${ }^{22}$ See Belletti 1999 for arguments that in Spanish a lexically specified subject is invariably external to the clause.

${ }^{23}$ The Boeckx 2001 account of resumptive pronouns purports to provide a unitary characterisation of resumption. However, it explicitly relies on a separation of "true" resumptive and "intrusive" pronouns, following Sells and Chao 1985, dismissing the optional resumptive use of pronouns in English:
} 
mechanisms distinct from regular processes. The only difference between pronouns which do allow update by node-merging processes and those which do not, is in the loss of the terminal-node restriction. Strong pronouns invariably retain such a restriction, behaving in all respect as regular lexical items. Clitic pronouns may have lost this restriction, though by no means all have done so. In all other respects, these pronouns function as regular anaphoric expressions, the loss of the restriction merely ensuring that they have a wider distribution.

With this mechanism of isolating one node and using that term as the basis for updating some antecedently established structure, we have a structural basis for focus effects. On the one hand, focus can be taken as an update to some propositional structure as already constructed in context (using prior context like the concept of topic):

(21) A: What did you eat for breakfast?

B: Porridge.

The short answer is an update device that directly modifies the contextually provided structure given by parsing the question. On the other hand, in canonical long-distance dependency structures such as (4), we have the structure where some term is presented as isolated on an initially unfixed node, with some open structure to which it is an update, both presented within a single construction process. So, like the concept of topic, focus is just an aspect of the dynamics of general utterance-interpretation. ${ }^{24}$

\section{At the Right Periphery}

The much less studied right periphery provides a new application area for these tools, where there are additional and much less well-understood restrictions. The challenge is whether the concepts of linked trees and unfixed

(i) The new guy from history who Sue says he's interested in Celtic studies is coming to the party.

But the Sells and Chao account which defines so-called intrusive pronouns as having a distinct E-type form of analysis, is highly problematic. E-type forms of interpretation, in which the appropriate witness for some set constitutes the denotation, by definition require a semantic computation over the entire clause which provides the antecedent, and are simply unavailable at the level of providing an interpretation of the pronoun itself . In relying on their analysis, the Boeckx analysis is at best incomplete.

${ }^{24}$ As expected, some languages allow mixed effects, in which a left-dislocated constituent may be paired with a pronoun which is itself at a potentially long-distance remove from the site at which it is to be construed. This phenomenon has been observed in Hebrew, but it occurs also in English, combining both topic and focus strategies: (i) As for Shalom, he I think should be given the position. 
nodes can explain the rather different topic and focus effects at the latter stages of the interpretation process, and we argue they indeed do so.

\subsection{Pronoun Doubling}

A natural candidate at the right periphery for a LINK transition from some completed node of type $t$ onto one requiring type $e$ - the inverse of the left periphery effect - is the Pronoun Doubling construction, with its characteristic background topic effect, in which the right-peripheral term serves as a reminder (see Herring 1994, inter al.):

(22) She talks too fast, Ruth Kempson.

(23) He's an idiot, that man at the cashdesk.

(24) lo conosco, Giovanni him I know Giovanni

[Italian]

(25) Maria lo compró, un coche Maria it bought, a car

[Spanish]

In these structures, in virtue of the LINK relation, an anaphoric expression must be identified as co-referential with the formula annotating the rightperipheral structure which is optional. This accounts directly for optionality

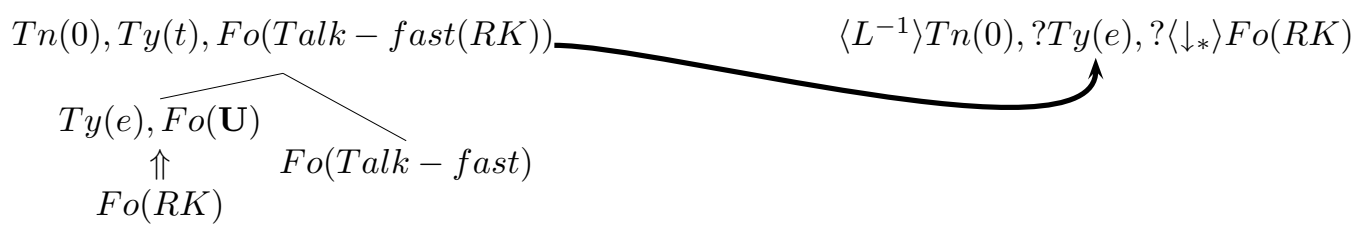

Figure 4: Parsing She talks too fast with LINK transition

of the post-posed noun phrase and the coreferentiality of it and the pronoun (22). The background topic effect is directly reflected in the defined sequence of actions. It arises because the pronoun is identified first as some contextually provided value and the right-peripheral expression as a decoration on the topnode of the linked structure has to agree with whatever value the pronoun has been assigned. The lack of availability of this type of reading at the left periphery is what we would expect, for at the left periphery the peripheral expression is interpreted as the context for subsequent identification of the pronoun; but at the right periphery, it is the other way round. The name is interpreted as having to be construed as being assigned 
the same term as already assigned as the construal of the pronoun. Hence its background topic effect, serving as a reminder. So we get our first taste of the asymmetry between left-right periphery effects derived from paying attention to the incremental dynamics of processing in context.

\section{2 "Rightward movement" effects}

Such postposed "topics" are not the only use of such right-peripheral expressions. ${ }^{25}$ Expressions postposed after the verb are regularly reported as having a contrastive use (Herring 1994 and many others) $:{ }^{26}$

$\begin{array}{lll}\text { (26) Tutie } & \text { wa-ss-ta } & \text { Chris } \\ \text { Eventually } & \text { come }_{P A S T, D E C L} & \text { Chris }\end{array}$

[Korean]

Eventually he came, Chris

This is not the end of the variability. In addition, in Italian, for example, Pinto 1998 reports that postposing of an indefinite subject NP buttresses a lack of specificity construal, the quantified expression thereby construed as taking narrow scope with respect to the tense specification:

(27) E' arrivato uno studente

is arrived one student

[Italian]

'One student has arrived'

In English, too, where the appearance of a subject in the preverbal position is obligatory, there is possible postposing to the post-verbal position of the associated complex subject expression, which appears to be yet another type of structure introduced late on in the interpretation process, that has to merge with the node already decorated by the pronoun:

(28) It is likely that Mary is wrong.

The occurrence of such expletives in English brings out the strictness of the left to right parsing process. Requirements on a node currently being parsed must be met by lexical action or by computational rule. The parse process cannot set aside words, if they happen not to fit the conditions imposed by the current node under development. Hence in English, like other non-subject-pro-drop languages, the existence of a particular form of

\footnotetext{
${ }^{25}$ Rightward movement within Minimalism is controversial, precluded by Kayne 1994 .

${ }^{26}$ There are multiple options in verb-final languages as to whether the nominal and doubling clitic are both marked with case, or both marked with a topic-marker, or only the pronominal so marked (see Kiaer in preparation).
} 
subject pronoun: without such a device, the parsing process would break down. Hence its role of projecting a place-holding metavariable and licensing moving on of the pointer. Yet such devices must be unlike regular anaphoric devices in one particular respect: they must have lost the restriction ensuring that decorate only a terminal node, for the metavariable they project must be able to be updated by arbitrarily complex structure. This is indeed the observable property of the expletive it in English (see figure 5). The

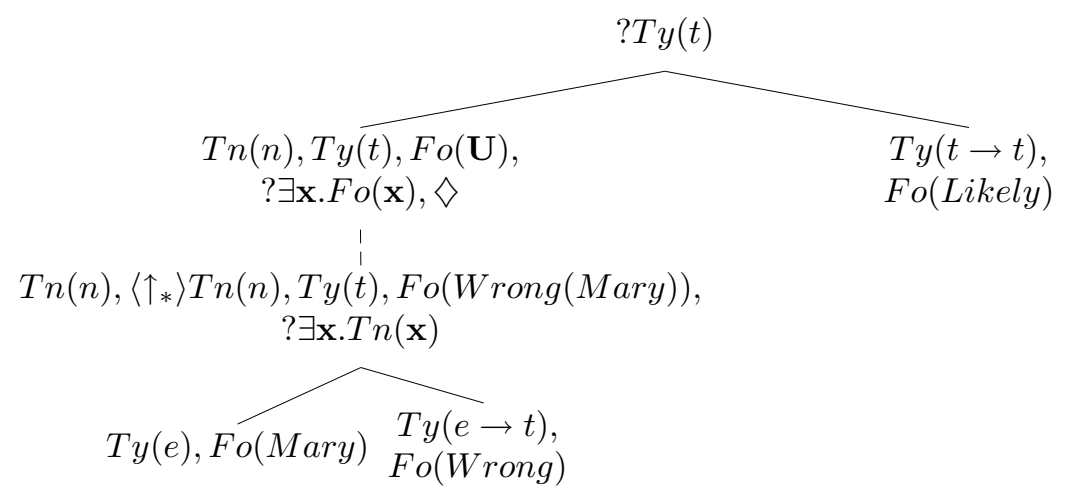

Figure 5: parsing It's likely that I'm wrong

essential right-roof constraint that is the corollary of these processes (Ross 1975) emerges from the dynamics of the left-right parse. When the pointer returns to the dominating type-t-requiring node, having licensed a shift in the parse to the predicate node, no decorations will be able to be compiled at the topnode since an outstanding requirement remains at that daughter node (reflecting strict compositionality on the tree itself). Accordingly the pointer will have to return to that node in order to license actions allowing that requirement to be met. A process of Late*Adjunction thus has to be invoked to enable an appropriate structure to be completed. ${ }^{27}$ This then provides the decoration, which, with a step of Merge unifying the two nodes satisfies the requirement which was otherwise preventing successful decoration of the mother node. The right roof constraint is thus an immediate consequence

\footnotetext{
${ }^{27}$ It might seem that a process of this type differs radically from *Adjunction as defined. However, this is a natural consequence of such late application, given formal properties of the update involved in resolving tree-node underspecification. Preparatory to such a step, the unfixed node is progressively percolated down through the tree, so that at the site of unification there is both the node with its open type-requirement, and the unfixed node. If the type matches, unification of nodes is possible. So a process of Late*Adjunction introducing an unfixed node of type $X$ from a node of matching type is already implicit in the framework. And at this late stage, when all structure is otherwise introduced, no other variant is available.
} 
of allowing a node to be but half-decorated, imposing the need of return to that node before decorations further up the tree can be compiled.

The result of licensing such a sequence of actions is that we have a mechanism which makes available late addition to skeletal structures lacking one possibly complex piece of structure to yield a new propositional whole. Following the pattern of extraposition, such late introduction of construal for the subject may be associated solely with buttressing some narrow scope effect (as in Italian). Given its presentation late on in the construction process, it may be taken to be a departure from what is in the context, hence contrastive. As we would expect, in languages in which the particular argument is licensed directly by the verb, a pro-drop structure, there will in consequence be processing ambiguity as to whether the postposed term is to be construed contrastively, via late application of *Adjunction, or as a background topic, via construction of a linked structure. And this is the reported situation for languages such as Spanish. As at the left periphery, the postposed subject may or may not be construed contrastively:

(29) Compró un coche, Maria

Bought a car, Maria

[Spanish]

She bought a car, Maria.

Just as there were mixed effects at the left periphery, so we expect the availability of mixed effects. In particular, we can combine left-periphery effects with right-periphery effects. So in Spanish, which displays the freest word order variation of the Romance languages, (30) is wellformed:

(30) Un coche compró, Maria

A car bought Maria

MARIA bought A CAR

Such examples, notably, involve a contrastive interpretation, and they confirm the analysis proposed. Within the framework, all partial trees along the process of growth must be wellformed, with all nodes uniquely identifiable, and this precludes the presence in any partial tree of more than one node analysed as unfixed at any one time. But, as we would expect in (30), the node decorated by un coche, which is introduced as unfixed, has been assigned a determinate position by the time that the node decorated by Maria is introduced through late*Adjunction. So (30) is wellformed, a legitimate means of conveying multiple focus interpretations. The presentation of un coche is taken to decorate a node to be treated as a subsequent update for the propositional structure to be set up in parsing compró, but so too is Maria. Hence the availability of a multiple focus interpretation. 


\section{Conclusion}

Across a range of left and right-periphery effects, we have shown how the mechanisms of building linked structures and unfixed nodes jointly provide a basis not merely for modelling familiar topic and focus effects at the left periphery, but also at the less-studied right periphery. The explanation has the bonus of explaining the right-roof constraint without special stipulation. More centrally to this paper, there is no superimposed independent level of information structure. All that is necessary to articulate concepts of context and information update which constitute the underpinnings to structural concepts of topic and focus is the exposition of the dynamic architecture intrinsic to natural-language processing.

\section{$6 \quad$ References}

Adger, D. and Ramchand, G. forthcoming. Revisiting wh dependencies: Merge and Move. In J.Rooryck and L.Cheng (eds.) Cambridge: MIT Press. Aissen, J. 1992. Topic and Focus in Mayan. Language 1992. 68.1 43-80.

Anagnastopoulou, E., Reimsdijk, H. van, Zwarts, F. (eds.) 1997. Materials on Left Dislocation. Amsterdam: John Benjamins.

Belletti, A. 1999. "Inversion" as focalization. CWPL 7, 9-45.

Blackburn, S. and Meyer-Viol, W. 1994. Linguistics,logic, and finite trees. Bulletin of Interest Group of Pure and Applied Logics 2, 2-39.

Boeckx, C 2001. Mechanisms of Chain Formation. Connecticut Ph.D

Büring. D. 1997. The meaning of topic and focus: The 59th Street Bridge Accent. Routledge.

Cann, R, Kaplan, T and Kempson, R. 2004. Data at the Grammar-Pragmatics Interface: the case of resumptive pronouns in English. In R.D. Borsley (ed.), special volume of Lingua.

Chomsky, N. 1965. Aspects of the Theory of Syntax. Cambridge MA: MIT Press.

Chomsky, N. 1971. 'Deep structure, surface structure, and semantic interpretation', in D. Steinberg and L. Jacobovits (eds) Semantics- An Interdisciplinary Reader, Cambridge. Cambridge University Press, 193-216.

Cinque, G. 1991. Types of A'-Dependencies, Cambridge, MA: MIT Press.

Erteshek-Shir, N. 1997. The Dynamics of Focus structure Cambridge: Cambridge University Press.

Fodor, J.A. 1981. Modularity of Mind. Cambridge MA: MIT Press.

Halliday, M. 1967. 'Notes on Transitivity and Theme in English, Part II', 
Journal of Linguistics 3,199-244.

Hausser, R. 1989. Computation of Language. Berlin:Springer.

Herring, S. 1994. 'Afterthoughts, antitopics, and emphasis: the syntactization of postverbal position in Tamil' in Butt, M. King, T. Ramchand, G. (eds.) Theoretical Perspectives on Word Order in South Asian Languages,119152. CSLI.

Kaplan, R. and Zaenen,A. 1989. Long-distance dependencies, constituent structure, and functional uncertainty. In Alternative Conceptions of Phrase Structure, ed. M.Baltin and A.Kroch, 17-42. University of Chicago Press.

Kayne, R. 1994. The Anti-symmetry of Syntax. Cambridge MA: MIT Press. Kempson, R. 2003. Japanese scrambling as growth of semantic representation. ms. KCL, London.

Kempson, R. Meyer-Viol, W. and Gabbay, D. 2001. Dynamic Syntax: The Flow of Language Understanding. Oxford: Blackwell.

Pinto, M. 1997. Licensing and Interpretation of Inverted Subjects in Italian. Ph.D. Utrecht.

Ranta, A. 1994. Type-Theoretic Grammar. Oxford: Clarendon Press.

Rizzi, L. 1997.The fine structure of the left periphery. In Haegemann, L.(ed.) Elements of Grammar. Dordrecht: Kluwer.

Ross, J.R. 1967. Constraints on Variables in syntax. Ph.D thesis. MIT

Schwarzschild, R. 1999. GIVENness, AvoidF and other constraints on the placement of accent. Natural Language Semantics 7.

Sells, P. and Chao, W. 1985. On the interpretation of resumptive pronouns. NELS 13.

Sgall P., Hajicôva, E. and Panevová, J. 1986. The Meaning of Sentence in its Semantic and Pragmatic Aspects, Dordrecht:Reidel.

Sperber, D. and Wilson, D. 1995. Relevance: Cognition and communication (2nd edition). Oxford: Blackwell.

Suñer, M. 1988. The role of Agreement in clitic-doubled constructions. NLLT 6.

Vallduvi, E. 1991. The informational Component. Ph.D. Pennsylvania. 